
\title{
3 Research Square \\ TGF- $\beta 2$ Levels in the Aqueous Humor are Elevated in the Second Eye of High Myopia Within Two Weeks After Sequential Cataract Surgery
}

\section{Weijia Yan}

Heidelberg University Hospital

\section{Yaping Zhang}

Xi'an Fourth Hospital, Shaanxi Eye Hospital, Affiliated Xi'an Fourth Hospital, Northwestern Polytechnical University

Hong Yan ( $\square$ yan2128ts@hotmail.com )

Xi'an Fourth Hospital, Shaanxi Eye Hospital, Affiliated Xi'an Fourth Hospital, Northwestern Polytechnical University

\section{Research Article}

Keywords: TGF- $\beta 2$, aqueous humor, cataract surgery, cytokine, axial length

Posted Date: September 29th, 2021

DOl: https://doi.org/10.21203/rs.3.rs-927355/v1

License: (9) (1) This work is licensed under a Creative Commons Attribution 4.0 International License. Read Full License 


\section{Abstract}

Purpose: To evaluate the level of transforming growth factors (TGFs) in the aqueous humor (AH) in the second eye of highly myopic cataract patients after receiving sequential cataract surgery and to study the correction between TGFs and axial length (AL) in myopic eyes.

Methods: The study included 13 patients (26 eyes) with high myopia with cataracts and 10 non-myopic patients (10 eyes) with cataracts. AH samples were used to detect the levels of TGF- $\beta 1,2$, and 3 in both groups. An array was used for verification of TGF- $\beta$ expression in AH samples obtained from 26 eyes of highly myopic patients with cataracts and 10 eyes of non-myopic patients with cataracts. According to $A L$, the samples were divided into three groups: $A(A L<28 \mathrm{~mm}), B(A L=28-31 \mathrm{~mm})$, and $C(A L>31 \mathrm{~mm})$.

Results: The level of TGF- $\beta 2$ in the AH sample of the second eye was significantly higher than that in the first eye in high myopia cataract patients $(\mathrm{p}=0.0147)$. Compared with the non-myopic cataracts patient group, the highly myopic cataracts group had a significantly higher TGF- $\beta 2$ concentration in the $A H$ in the second eye $(p=0.0156)$. Both TGF- $\beta 1$ and TGF- $\beta 3$ were expressed at low levels and had no difference between the two groups. Axial elongation in high myopia did not show a correlation with the $\mathrm{AH}$ concentration of TGF- $\beta 2$ levels.

Conclusions: In patients with high myopia undergoing sequential cataract surgery, the $\mathrm{AH}$ of the secondsurgery eye had a higher level of TGF- $\beta 2$ than that of the first-surgery eye, and it also had a significantly higher TGF- $\beta 2$ concentration than that of the control eye. This implies that high myopia could cause changes in the microenvironment of the eyes, and a protective mechanism was created to prevent the second eye from undergoing a sympathetic immune response.

\section{Introduction}

Cataract surgery is one of the most common surgical procedures performed worldwide. In recent years, cataract surgery has increasingly become a refractive procedure [1]. With the aging of the population, the incidence of cataracts has increased significantly. Authoritative data show that the improvement in visual function and quality of life after binocular cataract surgery far exceeds that of monocular cataract surgery. Therefore, timely second-eye surgery is essential for patients [2].

Topical anesthesia is now a commonly used method in cataract surgery. It has multiple advantages, including immediate vision restoration, improved safety, and reduced patient anxiety [3]. However, studies have shown that patients undergoing cataract surgery in the second eye with topical anesthesia usually report increased pain compared to the first eye. Therefore, our group previously assessed differences in 33 cytokine levels in the aqueous humor $(\mathrm{AH})$ of bilateral eyes receiving sequential cataract surgery. Transforming growth factor - beta2 (TGF- $\beta 2$ ) presented the highest concentration among the 33 detected cytokines. However, studies that only focused on changes in cytokines in $\mathrm{AH}$ in cataract patients with normal eye axial compression and concluded that the levels of TGF- $\beta 2$ in the second eye were higher than those in the first eye [4]. There is a lack of change in eyes with long axial lengths (AL). 
Myopia has become a worldwide health issue that may affect 2.56 billion people by 2020 , and a projected 4.7 billion people will have myopia by 2050 , comprising $49.8 \%$ of the world population [5]. High myopia, which is usually defined as eyes with a refractive error $<-6.00$ diopters [D] or $\geq 26 \mathrm{~mm}$, is more common in Asian populations [6]. The prevalence in certain young Asian populations has recently been shown to be as high as $16 \%$, and evidence indicates that the prevalence is increasing [7]. It is well documented that high myopia leads to a greatly increased risk of ocular pathologies, such as retinal detachment, macular degeneration, and glaucoma [8]. However, the pathogenesis of high myopia is unclear. Substantial studies have focused on surveying changes in the sclera, retina, and choroid in highly myopic patients, while fewer studies have evaluated the AH of certain patients [7], particularly in eyes undergoing sequential cataract surgery.

High myopia is considered an inflammation-related disease [9], for example, due to retinitis pigmentosa, pseudoexfoliation, uveitis, diabetic retinopathy or choroidal neovascularization, etc. [10]. Simultaneously, capsular contraction syndrome is more common in eyes with high myopia cataract patients [11]. Therefore, eyes with myopia might have a distinct internal microenvironment. Previous studies have demonstrated the expression of several inflammatory cytokines in the AH of eyes with high myopia [12]. Zhang et al. tested 440 cytokines in the AH of eyes with high myopia and found that MMP-2 and ANG-1 levels were significantly increased, suggesting that compared to that in nonmyopic eyes, the protein concentration is higher and different in eyes with high myopia. Additionally, high myopia is considered a disease related to inflammation, and highly myopic eyes are considered to have a proinflammatory internal microenvironment, which may lead to a variety of inflammation-related complications [6]. However, TGF- $\beta 2$ is reported to be the key cytokine involved in epithelial-myofibroblast transdifferentiation [13]. In eyes with high myopia, elevated TGF- $\beta 2$ was also found to be involved in scleral remodeling [14]. Therefore, TGF- $\beta 2$ in AH changes correspondingly, suggesting that there may be sympathetic ophthalmic uveitis in the second eye after first-eye cataract surgery. This may help explain why second eye surgery is often more painful [1].

The development and progression of myopia are associated with marked thinning of the sclera at the posterior pole, resulting in the extension of $A L$ and the occurrence of myopia. Major changes include reduced collagen synthesis, increased collagen degradation, reduced glycosaminoglycan synthesis, altered integrin expression, and increased fibroblast to myofibroblast differentiation [15]. Studies have indicated that the major changes are mediated by alterations in the levels of TGF- $\beta 2$ [16]. Our team previously extracted AH samples from both eyes of nonhigh myopia cataract patients after sequential cataract surgery. Thirty-three cytokines were identified in AH samples, and the expression of TGF- $\beta 2$ in the second eye was higher than that in the first eye [4]. Cataract extraction in highly myopic eyes provides us with great opportunities to collect AH from selected patients [12]. Based on previous research, this study aimed to assess the levels of TGF- $\beta$ s in both eyes of high myopic cataract patients after receiving sequential cataract surgery and using the $\mathrm{AH}$ samples that our group previously collected to compare the levels of TGF- $\beta$ s in high myopia cataract patients to nonmyopia cataract patients. Besides, we examined the AH level of TGF- $\beta 2$ in patients with myopia or cataract with different ALs to investigate the relationship between TGF- $\beta 2$ and axial elongation. Through comparative analyses of the concentration of 
TGF- $\beta$ in cataracts (control) and high myopia (patients), it is possible to achieve a better understanding of the microenvironment of eyes with high myopia and the immunological mechanism.

\section{Materials And Methods 2.1 Selection of patients}

This prospective longitudinal study was approved by the local ethics committee of Xi'an People's Hospital, China (No 20200035). It was performed by the Declaration of Helsinki. Written informed consent was obtained from all patients. During the period from Jan. 2020 to July 2020, AH samples were collected from 13 patients with high myopia cataracts. Patients with $A L \geq 26 \mathrm{~mm}$ were included with high myopia [6]. AL was measured with an ophthalmology ultrasound system (IOLMaster, Zeiss, Germany). Systematic ophthalmological examinations were performed during the presurgical visit to exclude patients with a history of eye surgery or any known eye diseases, such as glaucoma, retinitis, uveitis, and progressive retinal diseases. Patients who received local medications or underwent laser treatment were also excluded. From the day of admission to two weeks after surgery, all patients were asked to use $0.3 \%$ levofloxacin. All cataract surgeries were performed by the same surgeon $(\mathrm{YH})$ under topical anesthesia using phacoemulsification with intraocular lens implantation.

\subsection{Aqueous Humor Sample Collection}

Samples were obtained at the beginning of clear lens extraction or cataract extraction surgery. A 27gauge needle was used to aspirate $\mathrm{AH}$ samples (100-200 $\mu \mathrm{l})$ from the central pupillary area without touching the iris, lens, or corneal endothelium to avoid trauma and contamination of $\mathrm{AH}$ samples. Specimens were placed inside sterile Eppendorf tubes that were immediately stored below $-80^{\circ} \mathrm{C}$ until analysis.

\subsection{Analysis of TGF- $\beta(1,2$, and 3$)$ concentrations in $A H$ samples}

All analytical procedures were performed according to the manufacturer's instructions. Proprietary techniques were used to internally color-code microspheres with two fluorescent dyes. After concentrating the dyes, each dye was coated with a specific capture antibody. Next, an analysis from a test sample was captured by the bead to introduce the detection antibody. A Luminex ${ }^{\circledR}$ analyzer (MAGPIX®) was used to identify each microsphere, and the result of its bioassay was quantified based on fluorescent reporter signals. EMD Millipore combines the streamlined data acquisition power of Luminex ${ }^{\circledR}$ XPONENT ${ }^{\circledR}$ acquisition software with the sophisticated analytic capabilities of the new MILLIPLEX® Analyst 5.1, integrating data acquisition and analysis seamlessly with all Luminex® instruments.

\subsection{Statistical analysis}

D'Agootino-Pearson, Kolmogorov-Smirnov, and Shapiro-Wilk tests were employed to assess whether the data were normally distributed. If the criteria for normal distribution were satisfied, a paired $t$-test was 
used for comparison; if not, the nonparametric Wilcoxon signed-rank test was adopted. The student's $t$ test was used to detect differences between the first- and second eye surgery groups. Independentsample $t$-tests were used to detect differences between the second eye surgery and control groups. The Pearson correlation test was used to analyze the relationship between aqueous AL and TGF- $\beta 2$ concentrations. All analyses were performed using GraphPad Prism software version 8.0 (GraphPad Software, San Diego, CA). A two-tailed $\mathrm{P}<0.05$ was considered statistically significant for this analysis.

\section{Results}

\subsection{Baseline characteristics of all cataract patients}

A total of 16 patients with TGF- $\beta(1,2$, and 3$)$ data were enrolled, of which 3 cases did not meet the inclusion criteria and were excluded. A total of 13 patients (26 eyes) met the inclusion criteria and were included in the study. The preoperative distributions of age, sex, surgical eye, depth of the anterior chamber, eye-axial length, and interval time in patients with high myopia cataracts are summarized in Table 1. The two groups did not differ by age, sex, or time interval. The other parameters were significantly different between the two groups. Table 2 shows the statistics of best-corrected visual acuity, the visual acuity without correction, and spherical equivalent diopter of both eyes during the preoperative time and 1 week and 1 month after surgery.

Table 1. Baseline characteristics of patients between the two eyes' surgery

\begin{tabular}{ccccccc}
\hline Group & Age & Sex & Surgical eye & $\begin{array}{c}\text { AC depth } \\
(\mathbf{m m})\end{array}$ & $\begin{array}{c}\text { AL } \\
(\mathbf{m m})\end{array}$ & $\begin{array}{c}\text { Interval time } \\
\text { (d) }\end{array}$ \\
\hline $\begin{array}{c}\text { High myopia first } \\
\text { eye }\end{array}$ & & F 6 (30.76\%) & $\begin{array}{l}\text { OS:5 } \\
\text { OD:8 }\end{array}$ & $3.57 \pm 0.41$ & $30.53 \pm 2.59$ & \\
$\begin{array}{c}\text { High myopia second } \\
\text { eye }\end{array}$ & $54.62 \pm 7.24$ & M 7 (69.23\%) & $\begin{array}{l}\text { OS:8 } \\
\text { OD:5 }\end{array}$ & $3.58 \pm 0.42$ & $29.93 \pm 2.87$ & $11.08 \pm 3.60$ \\
\hline
\end{tabular}

AC: anterior chamber; AL: length of axial.

Table 2. Peri- and postoperative values of the two eyes' surgery. 


\begin{tabular}{cccc}
\hline First - eye & Preoperative & Postoperative $(\mathbf{1}$ w) & Postoperative (1 m) \\
\hline VAsc & $1.57 \pm 0.49$ & $0.48 \pm 0.19$ & $0.39 \pm 0.17$ \\
BCVA & $0.89 \pm 0.46$ & $0.17 \pm 0.16$ & $0.12 \pm 0.12$ \\
SE (diopter) & $-19.31 \pm 7.69$ & $-2.25 \pm 0.69$ & $-1.94 \pm 0.69$ \\
\hline Second - eye & Preoperative & Postoperative & \\
\hline VAsc & $1.46 \pm 0.51$ & $0.51 \pm 0.21$ & Postoperative $\quad(\mathbf{1 ~ m})$ \\
BCVA & $0.65 \pm 0.40$ & $0.17 \pm 0.18$ & $0.40 \pm 0.22$ \\
SE (diopter) & $-16.37 \pm 3.48$ & $-2.65 \pm 0.72$ & $0.17 \pm 0.21$ \\
& & & $-2.40 \pm 0.51$ \\
\hline
\end{tabular}

BCVA: best corrected visual acuity (LogMAR); VAsc: visual acuity without correction (LogMAR); SE: spherical equivalent.

\subsection{Comparison of the levels of TGF- $\beta(1,2$, and 3$)$ in AH between the first and second eyes}

In the present study, we obtained the levels of TGF- $\beta(1,2$, and 3$)$ in the AH of both eyes in high myopia patients after sequential cataract surgery. The results showed that there were significant differences in the levels of TGF- $\beta 2$ between the first and the second eye after surgery ( $P=0.0147$, paired Student's $t$-test). The level of TGF- $\beta 2$ in the second eye and control group was also significantly different $(P=0.016$, unpaired Student's $t$-test). Figure 1 shows the dot plots for TGF- $\beta 2$ levels in both eyes. In contrast, the levels of TGF- $\beta 1(P=0.28, P>0.05)$ and TGF- $\beta 3(P=0.99, P>0.05)$ were low and did not exhibit any significant differences between the two eyes. The mean differences were 3.89 and 0.21 , respectively.

\subsection{Relationship between TGF- $\beta 2$ and AL}

AH samples were collected from 26 subjects, including 8 eyes with $A L \leq 28 \mathrm{~mm}$ (group A), 9 eyes with AL from 28-31 mm (group B), and 9 eyes with $A L \geq 31 \mathrm{~mm}$ (group $C$ ). The average TGF- $\beta 2$ concentration was $3672.2 \pm 765.3 \mathrm{pg} / \mathrm{Ml}$ (mean \pm standard deviation). TGF- $\beta 2$ concentrations in groups $\mathrm{A}, \mathrm{B}$ and $\mathrm{C}$ were $3640.4 \pm 627 \mathrm{pg} / \mathrm{mL}, 3855 \pm 896.3 \mathrm{pg} / \mathrm{mL}$, and $3837.2 \pm 783.3 \mathrm{pg} / \mathrm{mL}$, respectively. Differences in the mean concentrations of TGF- $\beta 2$ in the three groups showed no significant differences $\left(P_{A B}=0.94, P_{B C}=\right.$ $0.99, \mathrm{P}_{\mathrm{AC}}=0.95$ ). TGF- $\beta 2$ concentration had no correlation with $\mathrm{AL}$ (correlation coefficient $r=0.021, \mathrm{P}=$ 0.92 ) in any subject (Figure 2). 


\section{Discussion}

High myopia is one of the most common eye disorders of public health concern worldwide and is regarded as an inflammation-related disease [9]. Studies have provided experimental and clinical evidence to support the possible association between inflammatory cytokines and myopia development or progression [6]. Previous research has indicated that TGF- $\beta$ is an important factor in the development of the eye [4]. The secreted TGF- $\beta$ cytokine exists in three isoforms: TGF- $\beta 1$, TGF- $\beta 2$, and TGF- $\beta 3$ [17]. TGF- $\beta 1$ plays a key role in controlling the immune system by differentially acting on cells according to cell type and differentiation stage. TGF- $\beta 3$ regulates the formation of cell adhesion molecules and extracellular matrix and controls wound healing by regulating the movement of epidermal and dermal cells in the injured skin [14]. TGF- $\beta 2$ is regarded as the major inform in the eyeball. TGF- $\beta 2$ is a normal component of $\mathrm{AH}$ and the main isoform in the sclera [17]. An increased level of TGF- $\beta 2$ was detected in the $\mathrm{AH}$ of glaucoma eyes, and a decreased level of active TGF- $\beta 2$ was detected in the $\mathrm{AH}$ of uveitic eyes [17]. Besides, all three isoforms can regulate collagen synthesis in scleral fibroblasts, and TGF- $\beta 2$ is the most effective isoform [14]. Although TGF- $\beta 2$ is a key factor in the development of myopia, it is not clear what role TGF- $\beta 2$ plays in this process.

Our previous study showed that the concentration of TGF- $\beta 2$ in AH samples was greater in the second eye than in the first eye during sequential cataract surgery, but the patients were all in the normal $A L$ in both eyes [4]. However, studies have indicated that changes in the expression of TGF- $\beta 2$ are associated with the development of high myopia. Thus, it is necessary to study possible changes in the concentration of TGF- $\beta 2$ in the contralateral eye of high myopia patients. Our data suggest that TGF- $\beta 2$ concentrations in the contralateral eye with high myopia were higher than those in the first eye. Previous studies have found that TGF- $\beta 2$ plays a central role in organ development and homeostasis, regulating cell proliferation, differentiation, and apoptosis [14]. Therefore, the current study may imply a protective mechanism for patients with high myopia and cataracts, which can prevent the sympathetic immune reaction caused by first-eye cataract surgery [1]. Therefore, the elevated TGF- $\beta 2$ levels in the contralateral eye compared to the first eye may be a response to the first eye surgery, which means that the operation on the first eye could affect the production of TGF- $\beta 2$ in the AH of the second eye. Besides, our statistical analysis showed that the level of TGF- $\beta 2$ was positively higher in the second surgery eye of high myopia than in nonmyopia cataract patients $(p=0.0156, t=2.612, d f=23)$, indicating that the changes in TGF- $\beta 2$ are correlated with the development of high myopia. This result was consistent with our previous study, which showed that TGF- $\beta 2$ levels in the AH of the second eye were elevated during bilateral sequential cataract surgery [4]. We believe that the high concentration of TGF- $\beta 2$ in the AH samples from highly myopic eyes may be attributed to the unique intraocular microenvironment, since other areas of highly myopic eyes, such as the sclera and retina-retinal pigment epithelium-choroid, were also reported to express high TGF- $\beta 2$ [18]. However, although TGF- $\beta 2$ is a key factor in the progression of high myopia development, it is still unclear what role TGF- $\beta 2$ plays in this process.

Studies have shown that TGF- $\beta 2$ is increased in eyes with excessive elongation of AL [17]. Consistent with previous animal models, TGF- $\beta 2$ is increased in myopic scleral tissue, and studies showed that TGF- 
$\beta 2$ inhibits the growth of cultured human scleral fibroblasts [19]. Therefore, the concentration of TGF- $\beta 2$ may be related to the pathophysiological changes in the development of high myopia. We studied the Pearson correlation test between the AL of high myopia patients and TGF- $\beta 2$. The levels of TGF- $\beta 2$ were not correlated with $A L$ (correlation coefficient $r=0.021, P=0.92$ ).

There were also some limitations to this study that necessitate our results be interpreted with some caution. First, only TGF- $\beta$ was increased in the 33 cytokines through our previous study, so we focused on the concentration of TGF- $\beta$ in the current study. However, changes in other factors are not necessarily ruled out. Second, the number of highly myopic patients with cataracts was relatively small in our study. Thus, we were able to obtain more fundamental data for follow-up and investigate more highly myopic patients after cataract surgery in our future study. Third, the evaluation of preoperative and postoperative time is not sufficient, which may need to be improved. Although we considered the levels of TGF- $\beta 2$ with $\mathrm{AL}$, it did not exhibit any correlation with the AH concentration of TGF- $\beta 2$. However, one study using AH samples reported that the concentrations of TGF- $\beta 2$ in 65 patients were positively correlated with $A L$, which is in the range of 24-29 mm [17]. The difference in these results might be attributed to the sensitivity of the detection methods and the small number of samples, and the average AL exceeded 30 $\mathrm{mm}$, which was far longer than that in this study.

In conclusion, the results of the present study revealed that the concentration of TGF- $\beta 2$ in the AH samples was significantly higher in the second surgery eye than in the first surgery eye in high myopia cataract patients, and the high myopia group had significantly higher TGF- $\beta 2$ concentration in the second eye than the control group. This finding suggests that eyes with high myopia may have a special internal microenvironment that is recognized as an inflammation-related disease. Meanwhile, it has been suggested that during binocular surgery for high myopia cataract patients, the contralateral eye should be properly delayed for surgery time. Although it may cause problems of binocular balance, it might be able to avoid the complications caused by the increase in TGF- $\beta 2$ concentration caused by short-term secondeye surgery.

\section{Abbreviations}

TGFs: transforming growth factors; $\mathrm{AH}$ : aqueous humor; $\mathrm{AL}$ : axial length

\section{Declarations}

\section{Acknowledgements}

We gratefully acknowledge the research participants who contributed samples for this study.

\section{Authors' Contributions}

W.-J.Y. contributed to the literature search, drafted, and edited the manuscript. Y.-P.Z. obtained the data and performed the statistical analyses. H.Y. conceived and designed the study, provided administrative 
and technical support, and edited the paper for the important intellectual content.

\section{Funding}

The study was supported by the National Nature Science Foundation of China (No.81873674), and the Cooperation Fund of Xi'an Fourth Hospital ( $\mathrm{LH}-6)$. The funding body provides the necessary funding to conduct this study, such as study design, sample collection and analysis, and professional language editing.

\section{Availability of data}

The datasets included in this study are available from the corresponding author upon reasonable request.

\section{Statement of Ethics}

Our study was approved by the local ethics committee of Xi'an People's Hospital, China (No 20200035). It was performed under the Declaration of Helsinki. Written informed consent for participation in the study was obtained from all patients.

\section{Consent for publication}

Not applicable.

\section{Conflicts of Interest}

The authors have no conflicts of interest to declare.

\section{References}

1. X. J. Zhu, D. Wolff, K. K. Zhang et al., "Molecular inflammation in the contralateral eye after cataract surgery in the first eye," Investigative Ophthalmology \& Visual Science, vol. 56, no. 9, pp. 5566-7553, 2015.

2. H. Yan. Yan's opinion on the bilateral cataract surgery in 2019. Chinese Documentary Press, Beijing, China.

3. R. Ursea, M. T. Feng, M. Zhou et al., "Pain perception in sequential cataract surgery: comparison of first and second procedures," Journal of Cataract \& Refractive Surgery, vol. 37, no. 6, pp. 1009-1014, 2011.

4. Y. Chen, Y. Zhang, K. Sun et al., "Higher TGF- $\beta 2$ level in the aqueous humor of the second eye versus the first eye in the course of sequential cataract surgery," Ocular Immunology and Inflammation, vol. 28, no. 3, pp. 439-445, 2020.

5. B. A. Holden, T. R. Fricke, D. A. Wilson et al., "Global prevalence of myopia and high myopia and temporal trends from 2000 through 2050," Ophthalmology. vol. 123, no. 5, pp. 1036-1042, 2016. 
6. J. S. Zhang, J. D. Wang, G. Y. Zhu et al., "The expression of cytokines in aqueous humor of high myopic patients with cataracts," Molecular Vision, vol. 26, pp. 150-157, 2020.

7. X. M. Duan, Q. J. Lu, P. Xue, et al., "Proteomic analysis of aqueous humor from patients with myopia," Molecular Vision, vol. 14, pp. 370-377, 2008.

8. S. H Lee, E. J. Lee, T. W. Kim, "Comparison of vascular-function and structure-function correlations in glaucomatous eyes with high myopia," British Journal of Ophthalmology, vol. 104, no. 6, pp. 807-812, 2020.

9. C. P. Herbort, M. Papadia, P. Neri, "Myopia and inflammation," Journal of Ophthalmic \& Vision Research, vol. 6, no. 4, pp. 270-283, 2011.

10. X. J. Zhu, K. K. Zhang, W. W. He et al., "Proinflammatory status in the aqueous humor of high myopic cataract eyes," Experimental Eye Research, vol. 142, pp. 13-18, 2016.

11. U. Chomańska, P. Kraśnicki, E. Proniewska-Skretek et al., "Anterior capsule contraction syndrome after cataract phacoemulsification surgery," Klinika Oczna, vol. 112, no. 7, pp. 243-246, 2010.

12. J. S. Yuan, S. J. Wu, Y. W. Wang et al., "Inflammatory cytokines in highly myopic eyes," Scientific Reports, vol. 9, no. 1, pp. 3517, 2019.

13. I. M. Wormstone, S. Tamiya, I. Anderson et al., "Duncan G. TGF-beta2-induced matrix modification and cell transdifferentiation in the human lens capsular bag," Investigative ophthalmology \& visual science, vol. 43, no. 7, pp. 2301-2308, 2002.

14. M. O. Li, Y. Y. Wan, S. Sanjabi et al., "Robertson AK, Flavell RA. Transforming growth factor-beta regulation of immune responses," Annual Review of Immunology, vol. 24, pp. 99-146, 2006.

15. S. Mérida, M. V. Villar, A. Navea et al., "Imbalance Between Oxidative Stress and Growth Factors in Human High Myopia," Frontiers in Physiology, vol. 11, pp. 463, 2020.

16. X. J. Zhu, M. J. Chen, K. K. Zhang et al., "Elevated TGF-beta2 level in aqueous humor of cataract patients with high myopia: potential risk factor for capsule contraction syndrome," Journal of Cataract \& Refractive Surgery, vol. 42, no. 2, pp. 232-238, 2016.

17. Y. Jia, D. N. Hu, J. Zhou, “Human aqueous humor levels of TGF-b2: relationship with axial length,” Biomed Research International, vol, 2014, pp. 258591, 2014.

18. K. K. Zhang, X. J. Zhu, M. J. Chen, et al., "Elevated transforming growth factor- $\beta 2$ in the aqueous humor: a possible explanation for high rate of capsular contraction syndrome in high myopia," Journal of Ophthalmology, vol. 2016. pp. 5438676, 2016.

19. J. S. Kang, C. Liu, R. Derynck, "New regulatory mechanisms of TGF-beta receptor function," Trends in Cell Biology, vol. 19, no. 8, pp. 385-94, 2009.

\section{Figures}




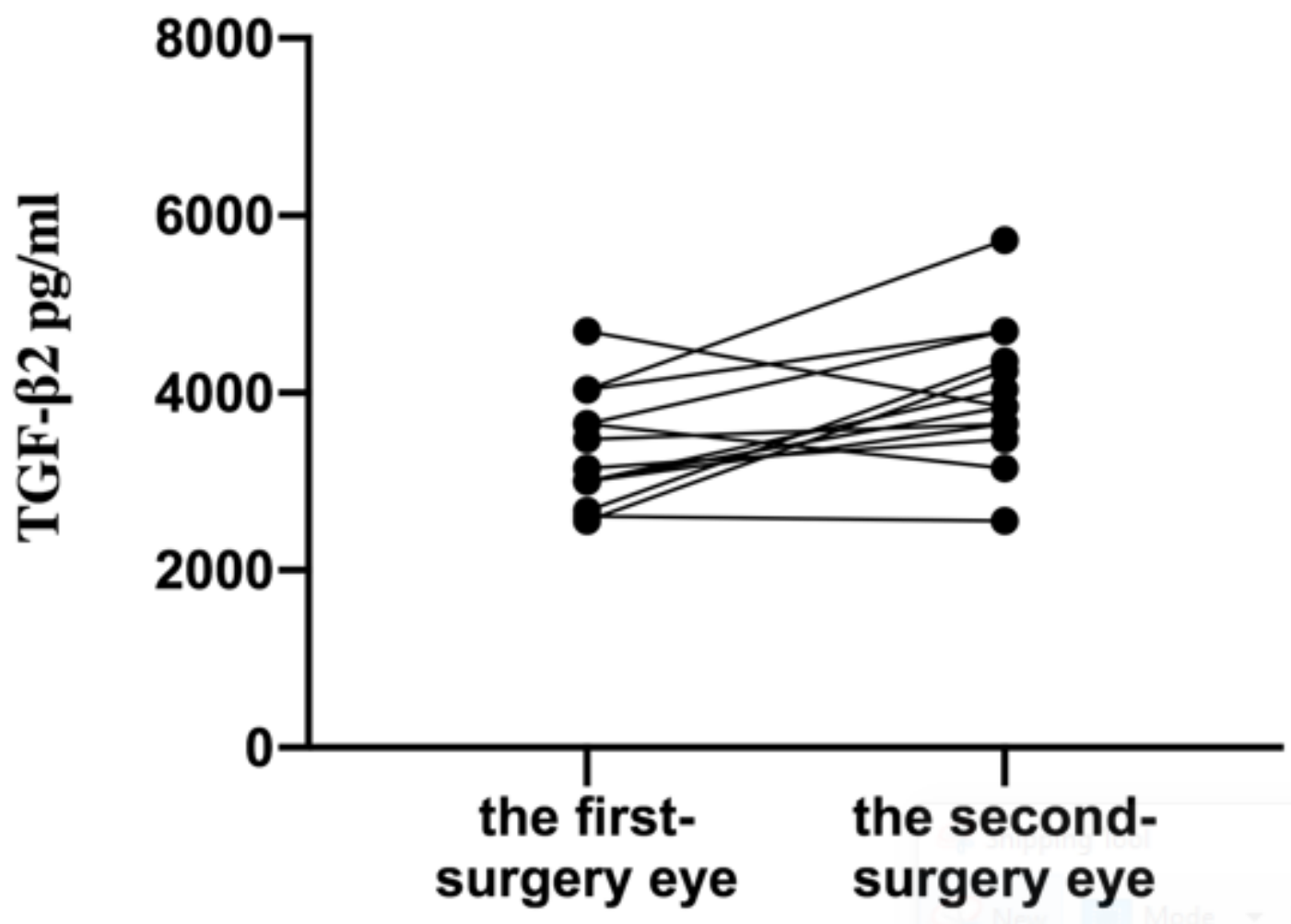

Figure 1

Paired dot plots showing the distribution of TGF- $\beta 2$ concentrations in the first and second eye $\mathrm{AH}$ samples. 


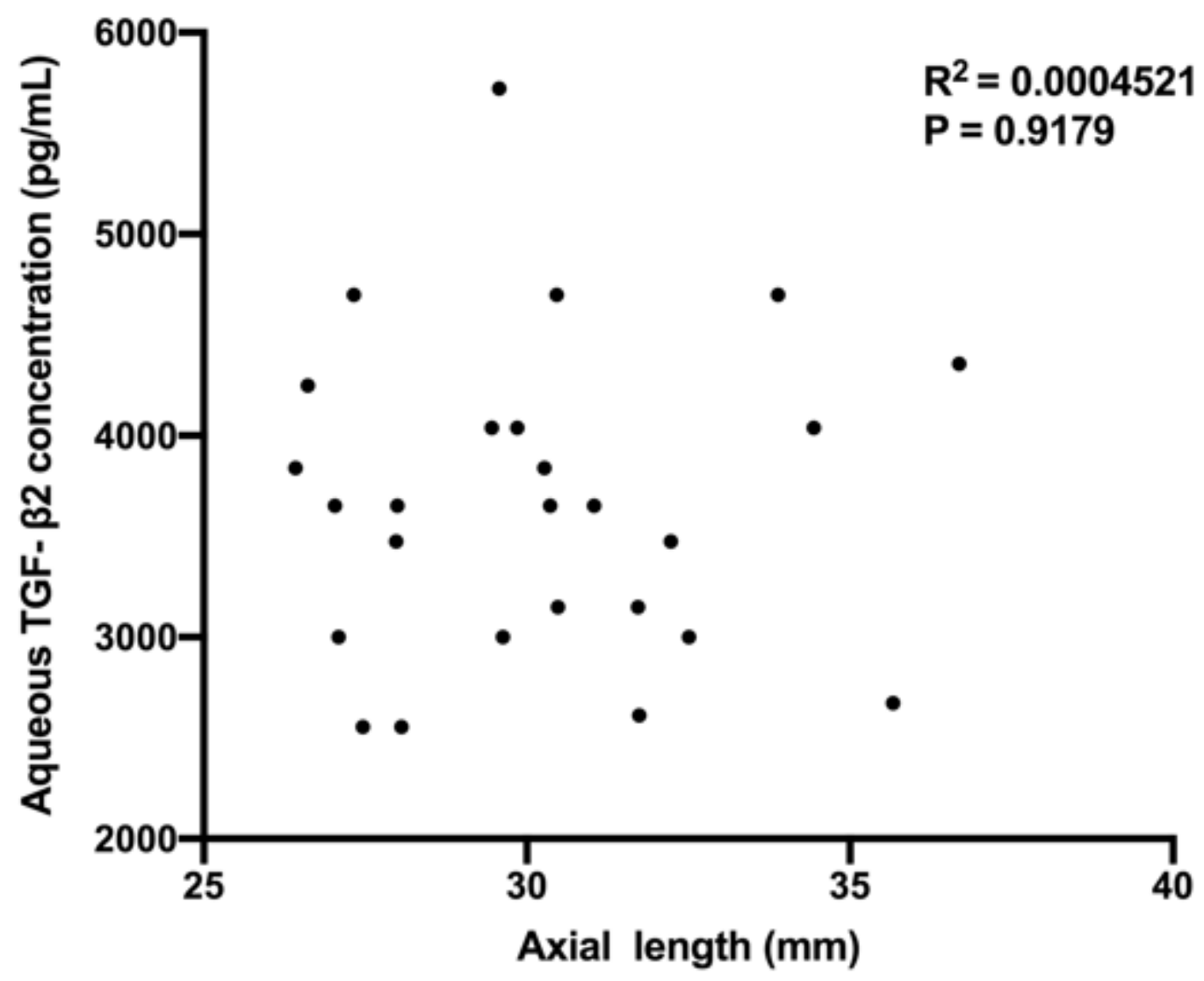

Figure 2

Correlation between AL and aqueous TGF- $\beta 2$. 